\title{
The Atacama Large Millimeter Array (ALMA)
}

\author{
Alwyn Wootten*National Radio Astronomy Observatory ${ }^{\dagger}$ \\ 520 Edgemont Rd, Charlottesville,VA 22901 USA
}

March 22, 2022

\begin{abstract}
The Atacama Large Millimeter Array, or ALMA, is an international telescope project which will be built over the coming decade in Northern Chile. With over $7000 \mathrm{~m}^{2}$ of collecting area comprised of 64 $12 \mathrm{~m}$ antennas arrayed over baselines up to $14 \mathrm{~km}$ in extent, ALMA will provide images of unprecedented clarity and detail. One revolutionary feature of ALMA will be its ability to combine interferometric and single telescope data, providing complete flux recovery. ALMA will cover a spectral wavelength range from $7 \mathrm{~mm}$ to $0.3 \mathrm{~mm}$ or shorter wavelengths, providing astronomy with its first detailed look at the structures which emit millimeter and submillimeter photons, the most abundant photons in the Universe.
\end{abstract}

\section{INTRODUCTION}

In this paper we review ALMA goals, the chosen site at Chajnantor, the scope of the project, and a few of the technical hurdles and proposed solutions, as well as the ALMA schedule.

\subsection{Goals: Science Aims and Drivers}

Several publications ${ }^{1,2}$ contain contributions describing the science goals of ALMA. In its basic formulation, the ALMA goal is to provide images of unprecedented clarity and detail in the millimeter and submillimeter spectral

${ }^{*}$ N. A. Project Scientist, ALMA

${ }^{\dagger}$ The National Radio Astronomy Observatory (NRAO) is a facility of the National Science Foundation operated under cooperative agreement by Associated Universities, Inc. 
range. This range contains two of the three primary peaks in the electromagnetic spectrum of the Universe; these are the two containing the preponderance of the observed energy in the Universe. The largest of these is the peak from the $3 \mathrm{~K}$ blackbody radiation relic of the Big Bang. That peak occurs in the millimeter range of the spectrum, as expected for any black body radiating at such a low temperature. The second strongest occurs at about $1.5 \mathrm{THz}$ or 200 microns wavelength. Light of these wavelengths cannot penetrate the atmosphere, as it is absorbed by water and other molecules-this maximum was identified only recently through satellite observations. Alas, with a satellite one is limited as to the size of telescope with which one can observe and anything we can currently put into space is far too small to give good images of the energy sources comprising this second peak. From its characteristic blackbody temperature of $\sim 20 \mathrm{~K}$ we are led to suspect that it is comprised of emission from the cold molecular clouds from which stars and planets in the Universe have formed, and from the young galaxies full of dust which host those molecular clouds. Recently, some telescopes have made progress in identifying the sources of this unknown radiation. Much of it-perhaps most of it-appears to come from tremendous episodes of star formation in galaxies at the earliest stage of their creation. Unfortunately, many sources of submillimeter radiation have not been identified optically, at least in part because their spectrum has been wholly redshifted to wavelengths blocked by the terrestrial atmosphere and in part because of tremendous amounts of dust endemic to the source, which absorb optical light and re-emit it at longer wavelengths. The only way to tell for sure is to get precise images of these sources. An instrument to provide these images must provide high resolution-matching that which will be available at other wavelengths, or from 0.01 " to 0.1 " and it must provide the sensitivity to invest these images with high dynamic range. ALMA has been defined to achieve these goals. It is currently under construction by an international partnership.

With construction funding begun in FY2002, ALMA will be built over the coming decade in Northern Chile. ALMA will be a revolutionary telescope, operating over the entirety of the millimeter and submillimeter wavelength band observable from the Earth's most lofty regions.

Table 1 summarizes the specifications for ALMA. The ALMA specifications are described in more detail in the ALMA Construction Project Book, which like other project details may be found on the Worldwide Web at www.alma.nrao.edu. To fully enable the order of magnitude gain in resolution and two order of magnitude gain in sensitivity over existing instruments which are ALMA's goal, it will be located at an elevation of $5000 \mathrm{~m}$ on the 


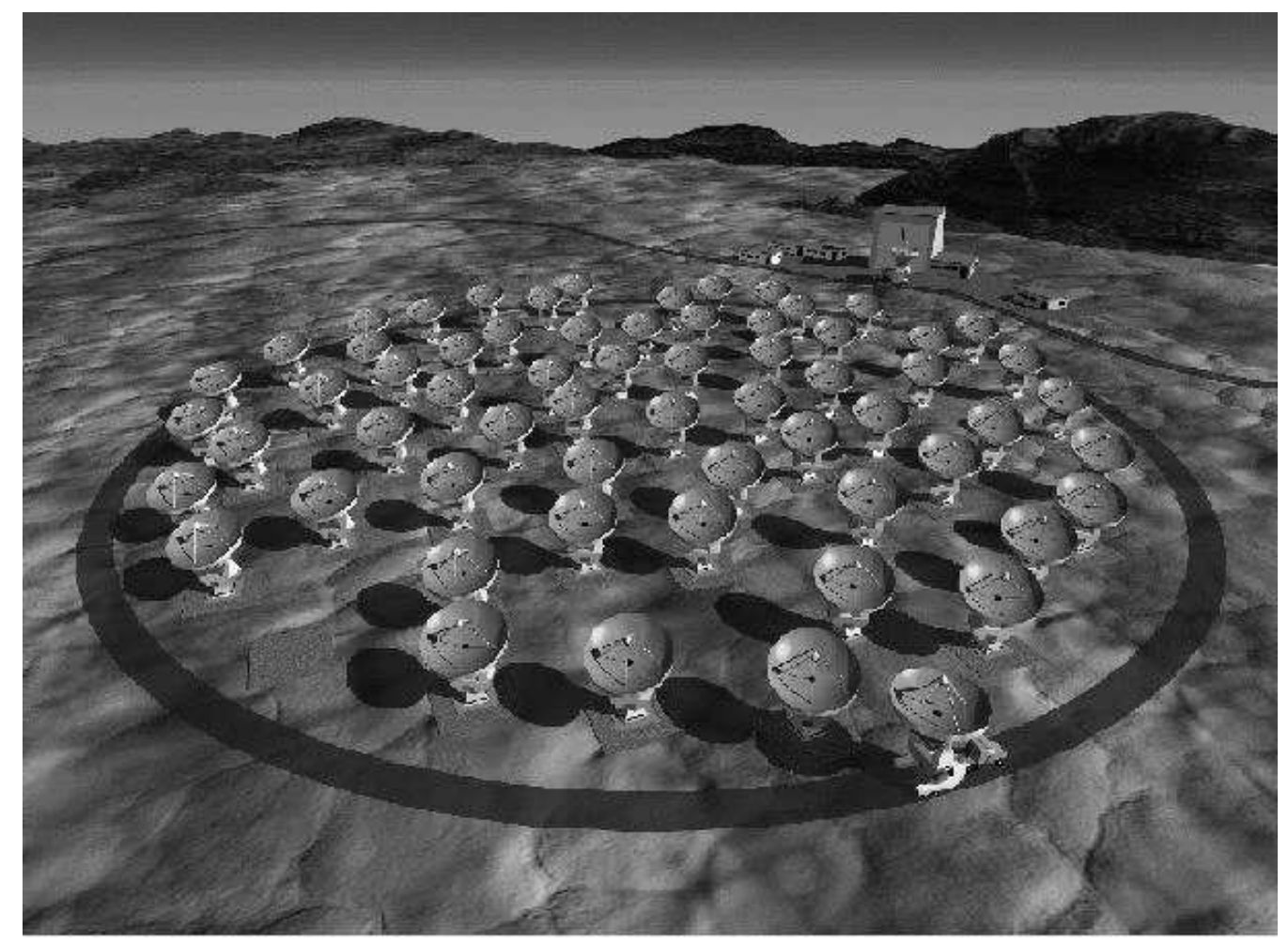

Figure 1: Artist's rendition of ALMA in its compact configuration. Image courtesy European Southern Observatory. 
Table 1: Summary of ALMA Specifications

\begin{tabular}{|l|r|}
\hline Parameter & Specification \\
\hline Number of Antennas & 64 \\
\hline Antenna Diameter & $12 \mathrm{~m}$ \\
\hline Antenna Surface Precision & $<25 \mu \mathrm{m} \mathrm{rss}$ \\
\hline Antenna Pointing Accuracy & $<0 . " 6 \mathrm{rss}$ \\
\hline Total Collecting Area & $>7000 \mathrm{~m}^{2}$ \\
\hline Angular Resolution & $02 \lambda(\mathrm{mm})$ \\
\hline Configuration Extent & $150 \mathrm{~m}$ to $14 \mathrm{~km}$ \\
\hline Correlator Bandwidth & $16 \mathrm{GHz}$ per baseline \\
\hline Spectral Channels & 4096 per window \\
\hline Number of Spectral Windows & 8 \\
\hline
\end{tabular}

Chajnantor Alitplano near San Pedro de Atacama in northern Chile, as shown in Figure 1 and described below. Table 2 summarizes the sensitivity of ALMA in various modes and at various frequencies, given the atmosphere at the Chajnantor site. The calculations assume $1 \mathrm{~mm}$ of preciptable water vapor above the site, observation at 1.3 air masses ( $40^{\circ}$ zenith angle) and antenna performance as given in Table 1.

\subsection{ALMA Partners}

ALMA has gained widespread support as an instrument astronomy needs to develop astronomical ideas through observation in the twenty-first century. The project, previously known at the Millimeter Array (MMA), has been endorsed by both the 1991 and 2000 Astronomy and Astrophysics Survey Committees of the National Academy of Sciences (US) as among the highest priority items for astronomical facilities to be constructed. In France, at a colloquium at Arcachon in March 1998 organized by CNRS/INSU, French astronomers put construction of ALMA, in its interim form as the Large Southern Array (LSA), as highest priority for future instruments. In Canada, ALMA was identified as top priority by the National Research Council's Long Range Planning Panel on Canada's Future in Astronomy until 2015. Dutch astronomers likewise established ALMA as the top priority 
Table 2: Summary of ALMA Sensitivities

\begin{tabular}{|l|c|c|r|}
\hline Band no. & $\begin{array}{c}\text { Frequency } \\
\text { Range }(\mathrm{GHz})^{a}\end{array}$ & $\begin{array}{c}\text { Continuum } \\
(\mathrm{mJy} ; 60 \mathrm{~s})\end{array}$ & $\begin{array}{r}\text { Line }\left(1 \mathrm{~km} \mathrm{~s}^{-1}\right) \\
(\mathrm{mJy} ; 60 \mathrm{~s})\end{array}$ \\
\hline 1 & $31.3-45$ & 0.02 & 5.1 \\
\hline 2 & $67-90$ & 0.028 & 4.9 \\
\hline $3^{b}$ & $84-116$ & 0.027 & 4.4 \\
\hline 4 & $125-163$ & 0.039 & 5.1 \\
\hline 5 & $163-211$ & 0.52 & 5.9 \\
\hline $6^{b}$ & $211-275$ & 0.071 & 7.2 \\
\hline $7^{b}$ & $275-370$ & 0.120 & 10 \\
\hline 8 & $385-500$ & 0.34 & 26 \\
\hline $9^{b}$ & $602-720$ & 0.849 & 51 \\
\hline 10 & $787-950$ & 1.26 & 66 \\
\hline
\end{tabular}

a Frequency ranges as given in Specifications for the ALMA Front End Assembly, AEC Version 1, 18 October, 2000.

$b$ These 4 bands are the highest priority bands, the others are the second priority bands, as judged by the ALMA Scientific Advisory Committee.

for instrumentation in the coming decade. In the U.K., the Astronomy Vision Panel of PPARC identified ALMA as the highest priority medium-term project.

This large set of astronomical community endorsements has gained ALMA a number of international partners. North American ALMA partner institutions include the United States National Science Foundation, through its National Radio Astronomy Observatory facility operated by Associated Universities, Inc., and the Canadian National Research Council. NRAO works in cooperation with a University consortium including the Owens Valley Radio Observatory of Caltech and the Berkeley Illinois Maryland Association. European partners include the European Southern Observatory; The Centre National de la Recherche Scientifique (CNRS), France; The Max Planck Gesellschaft (MPG), Germany; The Netherlands Foundation for Research in Astronomy, (NFRA); Nederlandse Onderzoekschool Voor Astronomie, (NOVA); The United Kingdom Particle Physics and Astronomy Research 
Council, (PPARC); The Swedish Natural Science Research Council, (NFR); and the Oficina de Ciencia y Tecnologia and Instituto Geografico Nacional (IGN), Spain. Chile, as host nation for the ALMA project, participates through its presence on the ALMA Coordinating Committee, the ALMA Science Advisory Committee and by providing the excellent site high in the Andean Altiplano and support for it. The National Astronomical Observatory of Japan may join the ALMA consortium soon.

\subsection{Location: the Site at Chajnantor, Chile}

In May 1998 NRAO recommended construction of the MMA on a site in Region II of northern Chile which lies on a high plain at the foot of three ancient volcanic peaks, Cerro Toco, Cerro Chajnantor and Cerro Chascon. The site (longitude $67 \mathrm{~d} 45 \mathrm{~m} \mathrm{~W}$, latitude $-23^{\circ} 01^{\prime} \mathrm{S}$ )lies near the Tropic of Capricorn, about $50 \mathrm{~km}$ east of the historic village of San Pedro de Atacama, $130 \mathrm{~km}$ southeast of the mining town of Calama, and about $275 \mathrm{~km}$ ENE of the coastal port of Antofagasta. It lies close to the border with Argentina and Bolivia beside the paved Paso de Jama road into Argentina and gas pipelines connecting Argentina sources with Chilean mines. The mean elevation is about $5000 \mathrm{~m}(16400 \mathrm{ft})$. Several sites had been tested but as scientific interest increased for the highest frequencies, sites at the highest altitudes became favored. Testing of the Chajnantor site began in April 1995 and continues to the present, a joint effort of NRAO and the European Southern Observatory (ESO). The Nobeyama Radio Observatory (NRO) operates a similar testing facility nearby at Pampa la Bola. The testing operations continue with the involvement of the Chilean university community. The land is administered by the Chilean Ministry of National Assets, having been set aside as a protected region for science by Presidential decree.

Up-to-date details of the monitoring of a number of parameters critical to ALMA's success continue and current details from the instrumentation may be found at the ALMA web site. Some salient characteristics include: the median annual temperature is $-2.5^{\circ} \mathrm{C}$ with annual 50 th percentile winds of $10.4 \mathrm{~m} \mathrm{~s}^{-1}$. The average barometric pressure is only 55 percent of the value at sea level. Humidity averages $39 \%$ and ultraviolet radiation is about $170 \%$ that at sea level. Transparency at $225 \mathrm{GHz}$ has been monitored for several years; the 50th percentile zenith optical depth at this frequency is 0.061 corresponding to a column of precipitable water of a little more than $1 \mathrm{~mm}^{3}$. With such a low water column, observations are possible at the atmospheric windows covered by ALMA Bands 9 and 10 (see Table 2) for roughly half 
of the time. Direct observations of atmospheric transparency including the supraterahertz windows at 1.035, 1.3 and $1.5 \mathrm{THz}$ have been published ${ }^{4}$ showing transmission of up to $20 \%$ in the highest frequency window.

\subsection{Science}

The mission of ALMA is to produce detailed spectral line and continuum images of objects emitting radiation in the millimeter and submillimeter spectrum accessible from the surface of the Earth. These high dynamic range images, covering a wealth of spatial scales and featuring total flux recovery, will allow ALMA scientists to study the formation of galaxies, stars and planets and the distribution of the chemical precursors necessary for life. Specifically, ALMA will allow astronomers to address a number of topics of high interest.

As mentioned above, the photons comprising the visible/infrared and submillimeter portions of the spectrum arise from two distinct features in the overall spectrum of background radiation. Images of the sky in these two bands are therefore complementary. The distinct nature of the submillimeter sky far from the Galactic Plane is compellingly illustrated by comparing the Hubble Deep Field (HDF) imaged at optical or infrared wavelengths with a submillimeter view (SCUBA $850 \mu \mathrm{m}^{5}$ ). As redshift increases, the volume of the Universe surveyed increases rapidly; this fact combined with an increasingly bright spectrum being brought into the observing band by higher redshifts (the so-called K-correction- $S_{\nu} \propto \nu^{3-4}$ ) ensures that the brightest sources at submillimeter wavelengths are distant $(\mathrm{z}>1)$ dusty galaxies. Furthermore, a generally higher star formation rate in the earlier epochs ${ }^{6}$ adds to the dominance of high redshift objects in the submillimeter. Comparison of the expected blackbody spectrum of the ultraluminous starburst galaxy Arp 220 as seen at high redshifts with the frequency coverage and the sensitivity of ALMA suggests that The Atacama Large Millimeter Array should be able to detect Arp 220-like dusty starburst galaxies out to a redshift of 10 or more. Galaxies like the present day Milky Way can also be detected out to z beyond 3 .

Although continuum emission from dust holds great interest, ALMA will also provide spectral line images to detail the kinematics of the gas, its excitation and its chemical and isotopic composition throughout the history of the universe. In the submillimeter and millimeter bands, the gas and dust enveloping galactic nuclei may be imaged without the optical obscuration which affects optical or infrared observations; the kinematics may be measured on spatial scales less than 100 pc or so. Various redshifted spectral 
lines-for example, all rotational transitions of $\mathrm{CO}$ and infrared fine structure lines such as [C II], [C I], [N II] and [O I]-can be detected and studied to derive the molecular gas content and elucidate the character of star forming activity.

Millimeter/submillimeter astronomy has discovered most of the $>120$ known interstellar molecules. These molecules may be quite complex-up to 12 atoms have been found in those small dense regions whose imaging will be ALMA's strength. ALMA offers complete coverage of the available windows from a site of unprecedented atmospheric clarity. ALMA improves currently available spectral sensitivity by more than an order of magnitude (the weakest lines detected today are of order $1 \mathrm{Jy} \mathrm{km} / \mathrm{s}$ in strength; compare to the ALMA sensitivity in Table 2 above). But as sensitivity improves, understanding of an already crowded spectrum may not be possible without an accompanying increase in spatial resolution. This ALMA also improves, by nearly two orders of magnitude, reaching resolutions of 10 milliarcsec. Furthermore, the throughput, measured by the spectral bandwidth open to ALMA (16 GHz), exceeds by several times that available now. A recent spectral survey from the $12 \mathrm{~m}$ telescope revealed some 15,000 lines in one spectral window toward the seven sources covered (Turner, private communication); ALMA's potential is clearly tremendous.

As stars form from a mixture of these molecules and dust, disks remain to form planets. Most $\mathrm{T}$ Tau stars possess disks with masses from 0.1 to 0.001 solar masses and sizes of 100-500 AU. Similar masses and sizes of disks appear to be present in protostars, embedded in dense molecular cloud cores. As the disks evolve, the chemistry continues to evolve, adapting to local conditions. With resolution reaching 5AU in nearby (140 pc) systems, ALMA will observe the chemical makeup of the disks as a function of vertical height (the disk 'atmosphere', with ultraviolet processing important higher up and condensation onto icy dust grains important mid-plane) and of radial distance from the star. Jets driven by the young star generate shocks into a cocoon of material surrounding the jet, chemically processing that material. Dutrey ${ }^{7}$ has summarized ALMA's contribution to molecular disk studies; many molecules have been observed in the disks about $\mathrm{T}$ Tau stars such as DM Tau and GG Tau, including CN, HCN, HNC, CS, $\mathrm{HCO}+, \mathrm{C}_{2} \mathrm{H}$ and $\mathrm{H}_{2} \mathrm{CO}$, suggesting a photon-dominated chemistry; even at 4" (600 AU) resolution changes in chemical structure of disks may be noted (e.g. LkCa15; $\mathrm{Qi}^{8}$ ). With ALMA, these structures will be more sensitively imaged; even time-resolved changes should be distinguished. ALMA will provide the sensitivity and resolution necessary to probe the planet-forming disk midplane. 
Fairly complex molecules have been detected in the interstellar medium. The simple sugar glycolaldehyde, a building block for more complex sugars ribose and glucose, was recently detected. Detection of its chemically reduced form, the ten atom molecule ethylene glycol, has just been announced. Searches for the simplest amino acid, the ten atom molecule glycine $\left(\mathrm{NH}_{2} \mathrm{CH}_{2} \mathrm{COOH}\right)$ have proven negative, lacking sensitivity. Glycine has a complex spectrum, exhibiting about a dozen lines per $\mathrm{GHz}$ on average. The Green Bank Telescope offers the sensitivity and wide bandwidth for a renewed search; ALMA will add to these attributes directivity, reducing spectral confusion in the complex regions where large molecules congregate. Detection may also be possible of other prebiotic molecules, such as adenine or uracile.

Molecules formed in dense regions may become incorporated in subplanetary or planetary objects. In cold regions, dense gas becomes highly deuterated. Elevated deuterium levels have been observed in some disks, as well as in cometary comae, suggesting some dense gas maintains its identity as planetary systems form. Evidence from the solar system suggests that some organics remain, even in the inner solar system, as they have become incorporated into discrete bodies. Carbonate blebs in the ALH84001 Martian meteorite maintained a heterogeneous pattern of magnetization suggesting transfer of the meteorite from Mars to Earth did not result in heating above $40^{\circ} \mathrm{C}$, Even after incorporation into larger bodies, organics may be transferred between bodies. Hence a direct connection may exist between complex molecules observable by ALMA in interstellar space and the complex prebiotic molecules found in small condensed objects in the solar system.

In solar system objects, ALMA will obtain unobscured subarcsecond images. As an example, the flow of sulfur oxides from the volcanoes on Io may be imaged to differentiate their origins.

ALMA will be able to detect wobbling motion in stars caused by planets orbiting them. ALMA will be able to detect planets in the process of formation. ALMA will only marginally detect thermal emission from nearby extrasolar large planets. Although it will not be able to image even nearby Earthlike planets, it will be able to detect the interstellar chemicals which are available for the nourishment of early life.

As stars evolve, nuclear processes in their interiors results in a striated structure. As their energy sources change according to local physical conditions, the star must adjust hydrodynamically, often shedding shells of processed material into the interstellar medium which cool and form dust and molecules. ALMA will image these shells to reveal the isotopic and chemical gradients that reflect the chronology of invisible stellar nuclear processing. 
ALMA is the only instrument, existing or planned which provides the combination of sensitivity, angular resolution and frequency coverage to achieve these scientific goals. By providing high fidelity images at subarcsecond resolution ALMA will complement both the present generation of gound-based telescopes, such as VLT, Gemini and Keck and their successors, GSMT or CELT, and space telescopes such as the HST and NGST. ALMA's images of cool thermal emission complements optical/infrared data provided by these telescopes to enable astronomers to explore all of the peaks of the electromagnetic spectrum at similar resolution and high sensitivity.

\section{Technical challenges and proposed solutions, schedule}

\subsection{The Antenna: Mechanical Engineering at the Heart of the Array}

Each of the two major partners plus Japan have contracted for construction of a $12 \mathrm{~m}$ diameter prototype antenna meeting the specifications imposed by the scientific goals for ALMA. These prototype ALMA antennas will be delivered to The ALMA Test Facility at the NRAO's Very Large Array (VLA) site near Socorro, N.M., for tests to ensure it meets the very demanding specifications of this project. VertexRSI of Santa Clara, California is the U.S. contractor for one antenna; this antenna is currently being assembled for tests to begin in November 2002. A second prototype design will be delivered to the same site in the spring of 2003 by a European consortium including Alcatel and European Industrial Engineering. These two antennas will be tested in order to determine which design is most appropriate for ALMA; both designs are the property of the ALMA project. The successful design will become the production design, to be built by the winner of a bidding process. A third prototype, built under contract with Mitsubishi, is expected to be delivered through an arrangement with the National Astronomical Observatories of Japan in 2003.

The ALMA prototype antenna makes extensive use of carbon fiber reinforced plastic (CFRP) technology in order for the antenna to maintain a stable parabolic shape in the harsh thermal and wind environment characteristic of the ALMA site at the 16,500 feet. The antenna surface accuracy must be better than 25 microns to enable efficient observations at the very highest frequency. Much of the time, ALMA will image fields larger than the primary beam, which means that multiple pointings of the antenna will 


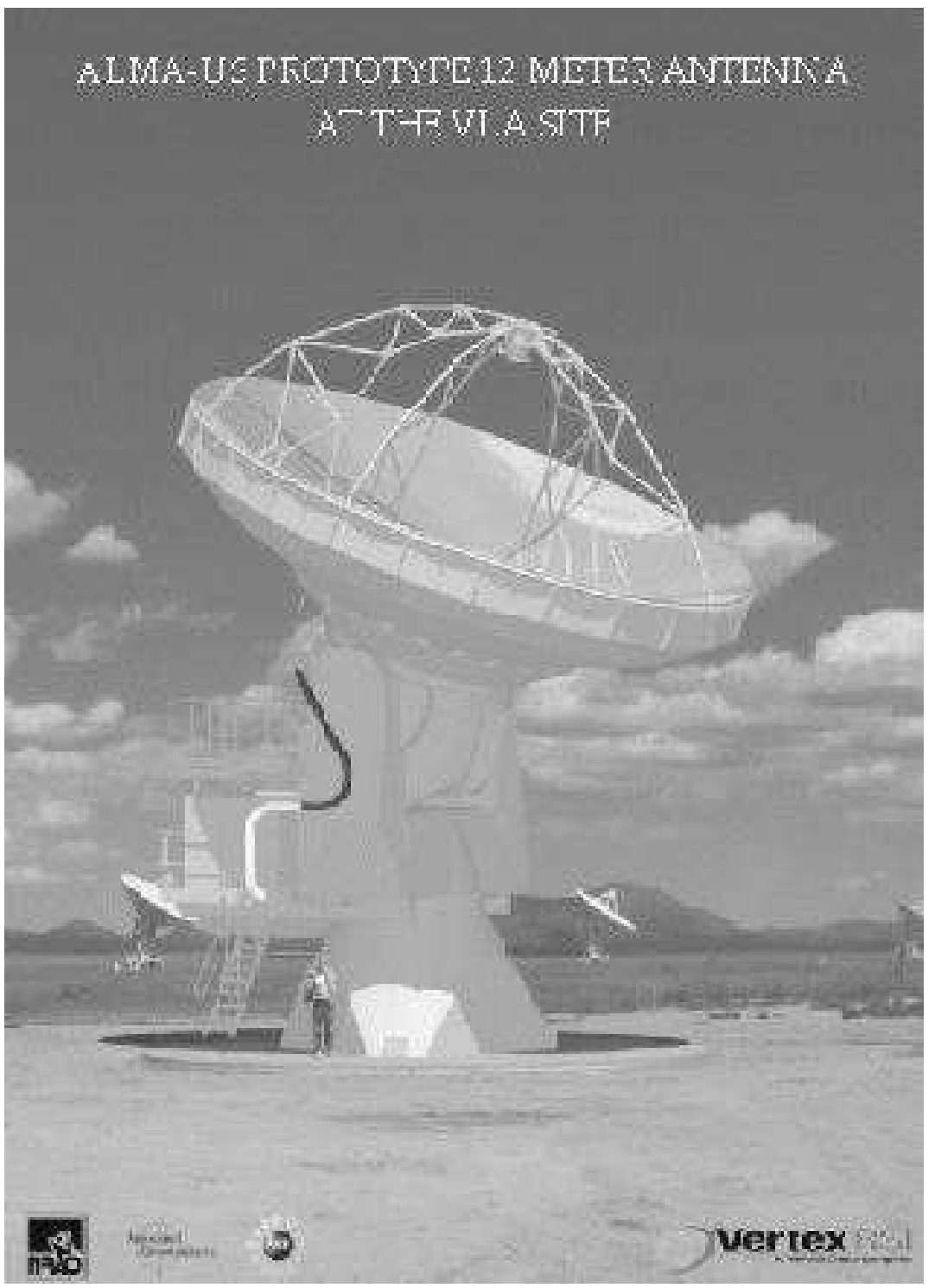

Figure 2: An engineering rendering of the 12 meter diameter prototype antenna that is being fabricated by VertexRSI to the very demanding ALMA specifications. The prototype antenna is under construction at the NRAO Very Large Array site for testing, as shown in this drawing. 
be fed into a single image which will include interferometric and total power data alike. For accurate imaging, the antenna and its calibration must be quite stable. For example, this requires that the antennas maintain a pointing accuracy better than 0.6 arcseconds, despite breezy conditions (50 percentile $6.5 \mathrm{~m} \mathrm{~s}-1$ ). The Chajnantor site affords no vegetative cover of consequence, so windblown grit and dust will occur and must not degrade the performance of the antenna. At such an altitude, temperature extremes occur-the annual median temperature is below freezing and can range \pm 20 $\mathrm{C}-$ yet antenna performance must be maintained. The ultraviolet radiation at this altitude is $170 \%$ of that at sea level, requiring construction of material robust to UV damage. All these factors provide a challenge to modern antenna design.

As the array is reconfigurable, the antennas must be transportable on an occasional basis by a transporter. The maximum baselines available are on the order of $14 \mathrm{~km}$. Current plans are to move antennas on a few-per-day basis along a self-similar configuration of roughly spiral geometry out to the largest configuration, where topography heavily constrains the design. This will provide images with a range of detail and a resolution of $0.015 " \lambda_{m m}$.

\subsection{The receivers: The world's largest superconducing ar- ray.}

Many laboratories worldwide contribute to the ALMA receiver effort. The receivers will cover the entire observable submillimeter/millimeter spectrum observable from ALMA's superb site. Eventually, ten receiver bands will span this region. Currently, receivers for the lowest two bands are planned to be transistor amplifiers. For frequencies above $\sim 80 \mathrm{GHz}$ the receiver designs employ superconducting tunnel junction mixers cooled to below $4 \mathrm{~K}$ with transistor amplifiers operating over a $4-12 \mathrm{GHz}$ or $4-8 \mathrm{GHz}$ range to provide the correlator with $16 \mathrm{GHz}$ of data. During initial construction, four receiver bands will be built and deployed as identified in Table 2. Orthogonal polarizations will be received, bringing ALMA's eventual total to over one thousand receivers-the most extensive superconducting electronic receiving system in astronomy. The complement is augmented by a system of radiometers operating at $183 \mathrm{GHz}$ which will monitor water vapor in the atmosphere above each telescope for path length correction of the incoming signals in a fashion analogous to the use of adaptive optics used on telescopes at visual and infrared wavelengths.

To cover the observable spectrum in ten bands, radiofrequency bandwidths up to $30 \%$ will need to be covered by receivers at some bands. Below 
$350 \mathrm{GHz}$, the receivers will be constructed to operate in single sideband to lessen atmospheric noise contributions.

To achieve this performance, the receivers will be housed in cartridges of a modular design,with all ten bands enclosed within a dewar of roughly $1 \mathrm{~m}$ diameter and stages cooled to 70, 15 and $4 \mathrm{~K}$. For each band, a modular cartridge will be developed which fits into the dewar from the bottom and which is held in place by flexible thermal links. Thus, all bands share the focal plane, and optics and calibration devices will sit atop the dewar.

\subsection{The Correlator: Achieving $1.6 \times 10^{16}$ multiply/add op- erations per second}

The ALMA Correlator will be located at the Chajnantor site. The analog input from the receivers on 64 antennas- 8 spectral windows, each of $2 \mathrm{GHz}$ bandwidth-will be digitized and transmitted at a rate of over 100 Gigabits per second from each antenna. The signal travels over fiber optic cables to digital filters, then to the correlator. The correlator must achieve 1.6 $\times 10^{16}$ multiply and add operations per second. It cross-correlates signals from $32 \times 63=2016$ pairs of antennas on 16 msec timescales; it also autocorrelates signals from 64 antennas on 1 msec timescales, producing 32 Gbyte s $^{-1}$ output. The correlator is currently under construction at the Central Development Laboratory of the NRAO on the University of Virginia grounds. Its design offers flexibility of selection of bandwidth, spectral window placement and a reasonably low power requirement of $150 \mathrm{~kW}$.

Correlator design and construction require a particularly long lead time; given the pace of technology prudence requires that ALMA consider the next generation correlator as the current generation correlator is constructed. Therefore, ALMA has fostered the design of the 'G2X Correlator' for possible deployment in the next decade. That design is currently scoped to provide twice as many channels in high dispersion mode as the ALMA Correlator; more than an order of magnitude more in low dispersion mode. Furthermore, the G2X Correlator concept improved sensitivity through threebit digitization in all modes rather than in the high dispersion modes only as provided by the ALMA Correlator. The concept envisions twice as many spectral windows (16) as the ALMA Correlator.

\subsection{Schedule}

The current ALMA Schedule is based upon the European construction phase beginning in mid-2002. The first antennas would then arrive in Chile some- 
time in late 2005. Commissioning observations would then begin late in 2006 and early science operations late in 2007. Under this schedule construction would be finished at the end of 2011 with full operations commencing in 2012 .

\section{$2.5 \quad$ References}

Alwyn Wootten \& F. Schwab Science with a Millimeter Array NRAO Workshop Vol. No. 14, Charlottesville: National Radio Astronomy Observatory (NRAO), edited by A. Wootten and F. R. Schwab, National Radio Astronomy Observatory, Green Bank, W. Va., 1988.

Alwyn Wootten Science with the Atacama Large Millimeter Array, ASP Conf. Ser. 235, edited by A. Wootten, Astronomical Society of the Pacific, San Francisco, 2001.

S. Radford \& R. Chamberlin "Atmospheric Transparency at $225 \mathrm{GHz}$ over Chajnantor, Mauna Kea, and the South Pole" ALMA Memo 334, 2000.

S. Matsushita, H. Matsuo, J. R. Pardo \& S. J. E. Radford "FTS Measurements of Submillimeter-Wave Atmospheric Opacity at Pampa la Bola II: Supra-Terahertz Windows and Model Fitting" Publ. Ast. Soc. Japan, 51 pp. 603-610, 1999.

Hughes, D. et al., "High-redshift star formation in the Hubble Deep Field revealed by a submillimetre-wavelength survey", Nature, 394 pp. 241-7, 1998.

Blain, A. W., Smail, I., Ivison, R. J. \& Kneib, J,, "The history of star formation in dusty galaxies", MNRAS, 302 pp. 632-648, 1999.

Dutrey, A. in Science with the Atacama Large Millimeter Array, ASP Conf. Ser. 235, edited by A. Wootten, Astronomical Society of the Pacific, San Francisco, pp. 215-224, 2001.

Qi, Chunhua, Aperture synthesis studies of the chemical composition of protoplanetary disks and comets, $\mathrm{Ph}$. D. Thesis, California Institute of Technology, 2001. 